\title{
Regulation of OLC1 protein expression by the anaphase-promoting complex
}

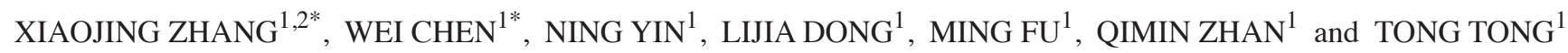 \\ ${ }^{1}$ State Key Laboratory of Molecular Oncology, Chinese Academy of Medical Sciences and Peking Union Medical College, \\ Cancer Institute and Cancer Hospital, Beijing 100021; ${ }^{2}$ Department of Oncology, Beijing \\ Chao-Yang Hospital, Capital Medical University, Beijing 100020, P.R. China
}

Received November 30, 2016; Accepted November 10, 2017

DOI: $10.3892 / \mathrm{ol} .2019 .9881$

\begin{abstract}
Overexpressed in lung cancer 1 (OLC1) is a potential oncogene overexpressed in human lung cancer and in other types of malignant tumor. The elevated expression of OLC1 contributes to tumor genesis and progression. However, the mechanisms regulating the expression of OLC1 remain unclear. In the present study, using lung and esophageal cancer cell lines, it was demonstrated that OLC1 was a short-lived, cell cycle-dependent protein regulated through the anaphase-promoting complex/cyclosome (APC/c)-ubiquitin pathway by directly interacting with the APC2 subunit. Through the action of two co activator proteins, cadherin 1 (Cdh1) and cell-division cycle protein 20 (Cdc20), the OLC1 protein was ubiquitinated and degraded. Following treatment with a proteasome inhibitor, OLC1 protein levels were elevated. Inversely, the upregulation of Cdh1 and Cdc20 facilitated OLC1 degradation. By inducing point mutations of the assumed degradation motif of OLC1, it was revealed that an intact destruction (D)-box was necessary. As expected, the D-box-mutated OLC1 exhibited a higher capacity for promoting cell growth and clone formation. Collectively, these findings indicate that the expression of the candidate oncogene OLC1 is cell cycle-dependent and
\end{abstract}

Correspondence to: Professor Tong Tong, State Key Laboratory of Molecular Oncology, Chinese Academy of Medical Sciences and Peking Union Medical College, Cancer Institute and Cancer Hospital, 17 Panjiayuan Nanli, Chaoyang, Beijing 100021, P.R. China

E-mail: tongtong@cicams.ac.cn

Dr Xiaojing Zhang, Department of Oncology, Beijing Chao-Yang Hospital, Capital Medical University, 8 Gongren Tiyuchang Nanlu, Beijing 100020, P.R. China

E-mail: cathyxjzhang@163.com

*Contributed equally

Key words: overexpressed in lung cancer 1, degradation, anaphase-promoting complex/cyclosome, cell-division cycle protein 20 , cadherin 1 , destruction box is regulated by an $\mathrm{APC} / \mathrm{c}$ mediated ubiquitin-proteasome pathway.

\section{Introduction}

The occurrence and development of malignant tumors is a complicated process, and numerous oncogenes and tumor suppressor genes are involved. Overexpressed in lung cancer 1 (OLC1) is a relatively novel candidate oncogene, which was originally discovered by Yuan et al (1) using suppression subtractive hybridization. It was identified that OLC1 was more highly expressed in squamous cell lung tumors compared with normal bronchial epithelial cells, and that it promoted tumor formation in nude mice. Similarly, it was also demonstrated that OLC1 was up regulated in human esophageal carcinoma and contributed to the proliferation of esophageal cancer cells (2).

The OLC1 gene was first designated as increased sodium tolerance 1 (IST1), which has also been identified and studied in non-lethal yeast mutants $(3,4)$. It encodes a protein that participates in the disassembly of endosomal sorting complexes $(5,6)$. It was reported that the human IST1 gene, now known as OLC1, may serve a significant function in cytokinesis in mitosis, as demonstrated in HeLa cells (7). As it is highly conserved and vital for organisms ranging from yeast to humans, studying the mechanisms that control OLC1 expression may provide a greater insight into its physiopathological function in human tumor genesis and progression.

A preliminary investigation indicated that OLC1 maybe degraded through the ubiquitin proteasome pathway and that cigarette smoke condensate (CSC) may affect this degradation (8). Through a bioinformatics analysis of the OLC1 protein, destruction (D)-box motif (amino acid sequence, RXXLXXXXN) was identified at amino acids 12-20. This motif is essential for the recognition and subsequent degradation of the protein by the anaphase-promoting complex/cyclosome (APC/c) $(9,10)$. As the destruction of APC targets is regulated by two activators, cell-division cycle protein 20 (Cdc20) and cadherin 1 (Cdh1) (11-13), these activators are also predicted to govern the degradation of OLC1. Previous studies regarding this topic predominantly focused on the effects of CSC on OLC1 stability (8), whereas the present study explores the in-depth mechanism by which OLC1 is degraded through the ubiquitin proteasome pathway. 
In the present study, it was revealed that OLC1 interacted with the APC/c through the APC2 subunit, assisted by two coactivator proteins, $\mathrm{Cdh} 1$ and $\mathrm{Cdc} 20$. The up regulation of Cdh1 and Cdc20 accelerated OLC1 degradation, whereas the down regulation of $\mathrm{Cdh} 1 / \mathrm{Cdc} 20$ resulted in OLC1 protein accumulation. In addition, the D-box sequence was characterized, as this motif was a critical determinant of OLC1 degradation. Mutations of the D-box motif enhanced OLC1 protein stability and induced an increase in cell growth and colony formation.

\section{Materials and methods}

Cell lines, cell culture and synchronization procedures. A stable OLC1-overexpressing cell line KYSE150/GFP-OLC1 and its null control cell line KYSE150/GFP were constructed as previously described (2). H1299 human lung carcinoma cells were purchased from the American Type Culture Collection (Manassas, VA, USA). KYSE150/GFP, KYSE150/GFP-OLC1 and H1299 cells were cultured in RPMI-1640 medium (Gibco; Thermo Fisher Scientific, Inc., Waltham, MA, USA) supplemented with $10 \%$ fetal bovine serum (FBS; HyClone; Thermo Fisher Scientific, Inc., Waltham, MA, USA), 100 U/ml penicillin, $100 \mu \mathrm{g} / \mathrm{ml}$ streptomycin and $400 \mu \mathrm{g} / \mathrm{ml} \mathrm{G} 418$ in a humidified atmosphere with $5 \% \mathrm{CO}_{2}$ at $37^{\circ} \mathrm{C}$.

To synchronize the cells at the $\mathrm{G}_{0}$ phase, the cells were incubated in RPMI-1640 medium without serum for $72 \mathrm{~h}$. The cells were transferred into fresh RPMI-1640 medium with serum for subsequent incubation. Then cells were collected every $2 \mathrm{~h}$.

For $\mathrm{G}_{2} / \mathrm{M}$ phase arrest, cells were incubated with 400 ng/ml nocodazole (Sigma-Aldrich; Merck KGaA, Darmstadt, Germany) for $16 \mathrm{~h}$. Following nocodazole treatment, cells were transferred into completeRPMI-1640 medium as described, and collected every $2 \mathrm{~h}$.

Reverse transcription polymerase chain reaction (RT-PCR). Total RNA was extracted from treated KYSE150/GFP cells with TRIzol reagent (Thermo Fisher Scientific, Inc.), and RNA $(6 \mu \mathrm{g})$ was reverse-transcribed according to the manufacturer's protocol (Super Script $^{\text {TM }}$ First-Strand Synthesis System for RT-PCR kit; Thermo Fisher Scientific, Inc., Waltham, MA, USA). For PCR amplification, $1 \mu \mathrm{l}$ cDNA solution was used as a template. The primer sequences used for the 435-bp OLC1 product were as follows: Forward, 5'-ACAGTGGGAGAGAGCACGTT-3' and reverse, 5'-GCACCTTGTCCTTTCTCTGC-3'. The sequences for the 299-bp GAPDH product were as follows: Forward, 5'-GCTGAGAACGGGAAGCTTGT-3' and reverse, 5'-GCC AGGGGTGCTAAGCAG-3'. The thermo cycling conditions for OLC1 are: $94^{\circ} \mathrm{C}$ for $5 \mathrm{~min}, 94^{\circ} \mathrm{C}$ for $30 \mathrm{sec}, 58^{\circ} \mathrm{C}$ for $30 \mathrm{sec}, 72^{\circ} \mathrm{C}$ for $1 \mathrm{~min}, 30$ cycles, and $70^{\circ} \mathrm{C}$ for $7 \mathrm{~min}$. The thermo cycling conditions for GAPDH are: $94^{\circ} \mathrm{C}$ for $4 \mathrm{~min}, 94^{\circ} \mathrm{C}$ for $30 \mathrm{sec}, 58^{\circ} \mathrm{C}$ for $30 \mathrm{sec}, 72^{\circ} \mathrm{C}$ for $30 \mathrm{sec}$ for $20 \mathrm{cycles}$, and $70^{\circ} \mathrm{C}$ for $4 \mathrm{~min}$.

Protein stability experiments. In order to identify the half-life of OLC1, the protein synthesis inhibitor cycloheximide (CHX; Sigma-Aldrich and Merck KGaA, Darmstadt, Germany, $100 \mu \mathrm{g} / \mathrm{ml}$ ) was added to the cell culture, and cells were collected at a range of time intervals. In order to ensure the effect of the proteasome on OLC1 degradation, MG132, a proteasome inhibitor, was added to the cell culture. The cells were incubated with $\mathrm{CHX}$ alone or with $\mathrm{CHX}$ and $20 \mu \mathrm{M}$ MG132 for a range of durations $(4,8,12$ and $16 \mathrm{~h})$, or with $\mathrm{CHX}$ and a range of MG132 concentrations (5, 10 and $20 \mu \mathrm{M})$. For each experiment, dimethyl sulfoxide (DMSO) was used as a control.

Immunoprecipitation and western blot analyses. Cells were harvested by washing twice with PBS and scraping away the cells, which were lysed in a lysis buffer on ice for $40 \mathrm{~min}$. The composition of the lysis buffer was as follows: PBS, $2 \mu \mathrm{g} / \mathrm{ml}$ aprotinin, $2 \mu \mathrm{g} / \mathrm{ml}$ leupeptin, $1 \%$ NonidetP-40 and $100 \mu \mathrm{g} / \mathrm{ml}$ phenylmethylsulfonyl fluoride. The lysates were harvested via centrifugation at $4^{\circ} \mathrm{C}$ at $16,000 \mathrm{x}$ g for $20 \mathrm{~min}$, and the supernatants were extracted to collect complete protein samples.

For immunoprecipitation, $500 \mu \mathrm{g}$ total cellular protein lysate was incubated with $\sim 2 \mu \mathrm{g}$ of the indicated antibodies, including OLC1 rabbit anti-human polyclonal antibody (ready for use), which was prepared and purified using the purified recombinant glutathione-S-transferase-OLC1 by Wuhan Sanying Biotechnology, Wuhan, China (1) and actin mouse anti-human monoclonal antibody (ready for use; cat. no., sc-8432; Santa Cruz Biotechnologies, Santa Cruz, $\mathrm{CA}, \mathrm{USA})$ at $4^{\circ} \mathrm{C}$ for $6 \mathrm{~h}$ and then incubated with $20 \mu \mathrm{l}$ A/G-agarose beads (Santa Cruz Biotechnology, Inc., Dallas, TX, USA) overnight at $4^{\circ} \mathrm{C}$. Next, immune complexes was harvested via centrifugation at $4^{\circ} \mathrm{C}$ at $1,000 \mathrm{x}$ for $5 \mathrm{~min}$ and washed 7-8 times with $1 \%$ Nonidet P-40. The immunocomplexed proteins were then subjected to a western blot analysis.

For the western blot analysis, 80-100 $\mu \mathrm{g}$ cellular protein was resolved via $10 \%$ SDS-PAGE and transferred to Immobilon-P polyvinylidene fluoride membranes (Millipore, Bedford, MA, USA). Then the membranes were incubated in PBS containing 5\% nonfat milk at room temperature for 30 min to block nonspecific binding. Next the membranes were incubated at $37^{\circ} \mathrm{C}$ for $2 \mathrm{~h}$ with the following antibodies: OLC1 (dilution, 1:100; Wuhan Sanying Biotechnology, Wuhan, China), APC2 (dilution, 1:500; cat. no., sc-517022), cyclin A (dilution, 1:500; cat. no., sc-271682), cyclinD1 (dilution, 1:500; cat. no., sc-246), cyclin E (dilution, 1:500; cat. no., sc-247), actin (dilution, 1:1,000; cat. no., sc-8432), GFP (dilution, 1:500; cat. no., sc-9996) (all from Santa Cruz Biotechnology, Inc., Dallas, TX, USA), Cdc20 (dilution, 1:2,000; cat. no., NB100-59828; Novus Biologicals, LLC, Littleton, CO, USA,) ubiquitin (dilution, 1:1,000; cat. no., U5379; Sigma Aldrich; Merck KGaA) and Cdh1 (dilution, 1:1,000; cat. no., C7855; Sigma Aldrich; Merck KGaA) and cyclin B1 (dilution, 1:250; cat. no., 554178; BD Biosciences, Franklin Lakes, NJ, USA). The membranes were washed in PBS with $0.1 \%$ Tween 20 five times and then incubated at room temperature for $1 \mathrm{~h}$ with a goat anti-mouse (cat. no., sc-2005) or goat anti-rabbit (cat. no., sc-2004) horseradish peroxidase-conjugated secondary antibody (the two antibodies were diluted at 1:1,000 and were purchased from Santa Cruz Biotechnology, Inc.). Next the membranes were washed five times, and antibody reactivity was visualized using a FUJIFILM LAS-4000 machine. Images were edited using Multi-Gauge Fujifilm (version 3; Fujifilm Life Science, Tokyo, Japan) and Photoshop CS software (Adobe Systems, Inc., San Jose, CA, USA). 
Cell protein ubiquitination assay. Cultured cells were treated with $20 \mu \mathrm{M}$ MG132 or DMSO. Then cells were collected andlysed on ice for $30 \mathrm{~min}$ with thelysis buffer. Then the lysates were centrifuged at $16,000 \mathrm{xg}$ for $20 \mathrm{~min}$ at $4^{\circ} \mathrm{C}$. In each immunoprecipitation reaction, as described previously, $500 \mu \mathrm{g}$ total cellular protein lysate was incubated with $10 \mu \mathrm{l}$ of the indicated antibody. Then the immune complexes were pulled down and analyzed with western blotting. Using an anti-ubiquitin antibody, the polyubiquitinated OLC1 protein was probed.

Plasmid mutation and cell transfection. pEGFP-N1 and pEGFP-N1-OLC1 plasmids were supplied by Professor Shujun Cheng of the Chinese Academy of Medical Sciences and Peking Union Medical College (Beijing, China). pCMV-Myc-Cdh1 and pCMV-Myc-Cdc20 plasmids were provided by Professor James Hsieh of Washington University (Seattle, WA, USA) and pCMV-3 plasmids were obtained from the State Key Laboratory of Molecular Oncology (Beijing, China).

In order to identify the specific sequences that acted as potential degradation signals for OLC1, a generated D-boxsite-directed mutation construct, pEGFP-N1-mut-OLC1, was produced in conjunction with Shanghai Gene Chem Co., Ltd. (Shanghai, China). For cell transfection, H1299 cells were seeded onto $35-\mathrm{mm}$ plates 1 day prior to transfection to allow cells to reach $95 \%$ confluence at the time of transfection. To prepare each plate, $10 \mu 1$ Lipofectamine ${ }^{\circledR} 2000$ (Invitrogen; Thermo Fisher Scientific, Inc.) and $4 \mu \mathrm{g}$ plasmid DNA were added to $500 \mu \mathrm{l}$ RPMI-1640 medium without antibiotics. The solutions were mixed lightly, left to stand for $20 \mathrm{~min}$ at room temperature, diluted with $3 \mathrm{ml}$ RPMI-1640 medium without antibiotics, and added to the plates to stand for $6 \mathrm{~h}$ at $37^{\circ} \mathrm{C}$. Next, $4 \mathrm{ml}$ RPMI-1640 medium from each plate was exchanged for an equal volume of RPMI-1640 medium supplemented with $10 \%$ FBS, and plates were incubated for an additional $48 \mathrm{~h}$. Cells were then harvested.

Small interfering RNA (siRNA) construction and cell transfection. siRNA for Cdh1 and Cdc20 were constructed by Jikai Biotechnology, Inc. The Cdh1 siRNA sequence was 5'-UGAGAAGUCUCCCAGUCAGdTdT-3' and the Cdc20 siRNA sequence was 5'-AAACCTGGCGGTGACCGCTAT-3'.

For cell transfection, H1299 cells were seeded onto 35-mm plates 1 day prior to transfection to allow the cells to reach $70 \%$ confluence at the time of transfection. To prepare each plate, $4 \mu \mathrm{g}$ siRNA and $10 \mu \mathrm{l}$ Lipofectamine ${ }^{\circledR} 2000$ (Invitrogen; Thermo Fisher Scientific, Inc.) were added to $500 \mu 1$ RPMI-1640 medium without antibiotics. The solutions were mixed gently, sitting for $20 \mathrm{~min}$ at room temperature, diluted with $3 \mathrm{ml}$ RPMI-1640 medium without antibiotics and added to the plates to sit for $6 \mathrm{~h}$ at $37^{\circ} \mathrm{C}$. Next, $4 \mathrm{ml} \mathrm{RPMI}-1640$ medium from each plate was exchanged for an equal volume of medium with $10 \% \mathrm{FBS}$, and plates were incubated for $48 \mathrm{~h}$. Cells were then harvested.

Cell proliferation assay and colony formation. Cells in the exponential growth phase were seeded at a density of 3,000 cells per well in 12-well plates, in triplicate. Cells were then counted every $24 \mathrm{~h}$ for 5 days to produce a growth curve. All experiments were repeated three times.
At $24 \mathrm{~h}$ after transfection, cells transfected with each specific plasmid were seeded in RPMI-1640 with 10\% FBS and $400 \mu \mathrm{g} / \mathrm{ml} \mathrm{G} 418$ at a density of 1,000 cells per well in six-well plates in triplicate. After 14 days, the cells were washed with PBS, fixed in cold methanol and stained with $0.5 \%$ crystal violet for $10 \mathrm{~min}$ at room temperature. Colonies with more than 50 cells were counted. All experiments were repeated three times.

Statistical analysis. SPSS version 11.5.0 (SPSS, Inc., Chicago, IL, USA) was used to perform all statistical analyses. A Student's t-test was used for comparison between two groups. The differences between multiple groups were analyzed using a one-way analysis of variance and Fisher's least significant difference test as a post hoc test. $\mathrm{P}<0.05$ was considered to indicate a statistically significant difference.

\section{Results}

OLC1 expression is cell cycle-dependent. To explore whether the expression of OLC1 is associated with cell cycle progression, two cell cycle synchronization approaches were adopted in order to detect the OLC1 protein expression in KYSE150/GFP cells. By means of serum starvation, cells were synchronized at the $\mathrm{G}_{0}$ phase. Cells were arrested at the mitotic phase with the microtubule inhibitor nocodazole. Cells were subsequently collected every $2 \mathrm{~h}$, and the expression of OLC1 protein was analyzed with western blot assays. The expression of cyclin B1, D1, A and E were analyzed to assess cell cycle progression. As presented in Fig. 1A, following serum starvation, OLC1 protein was highly expressed during most of the $\mathrm{G}_{0} / \mathrm{G}_{1}$ phase, remained at relatively low levels at the $S$ phase and demonstrated a slightly elevated level in the $\mathrm{G}_{2} / \mathrm{M}$ phase. Similar results were obtained in the experiments performed using no codazole to synchronize cells at the mitotic phase (Fig. 1B). In an additional experiment, the mRNA expression of OLC1 was examined, which demonstrated relatively little change throughout the whole cell cycle (Fig. 1C). In conclusion, these observations indicate that OLC1 protein expression is cell cycle-dependent and that it is primarily regulated via post-translational, and not transcriptional, modification.

OLC1 protein is short-lived and its stability is regulated by the ubiquitin proteasome pathway. Considering that the OLC1 protein expression was determined to be cell cycle-dependent, similar to the cyclins, the half-life of OLC1 protein was not expected to be long. Following treatment with the protein synthesis inhibitor $\mathrm{CHX}$ for a range of times, the half-life of OLC1 was analyzed. OLC1 protein expression decreased to $\sim 50 \%$ at $8 \mathrm{~h}$ of $\mathrm{CHX}$ treatment compared with the expression observed at $0 \mathrm{~h}$ and in the untreated control cells (Fig. 2A), suggesting that OLC1 protein has a rapid turnover rate. Given that $80-90 \%$ of intracellular proteins are degraded by the ubiquitin proteasome pathway, it was predicted that OLC1 protein stability may be regulated though this mechanism. Therefore, the selective proteasome inhibitor MG132 was used to treat KYSE150/GFP and KYSE150/GFP-OLC1 cells with or without $\mathrm{CHX}$, which was followed by an analysis of endogenous and exogenous OLC1 protein expression. The addition of different concentrations of MG132 resulted in 
A

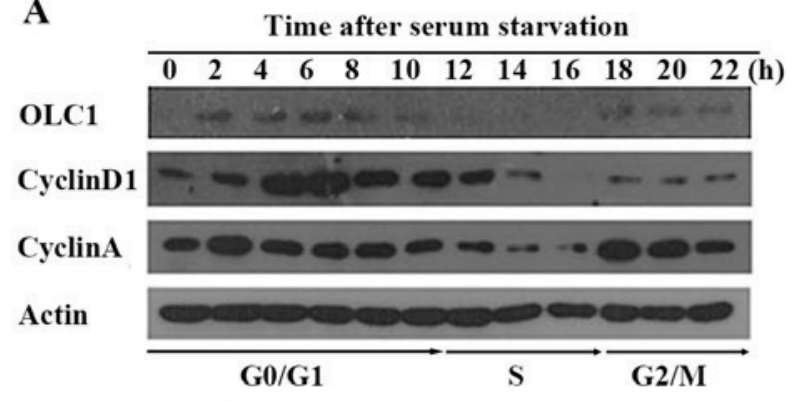

B

Time after nocodazole

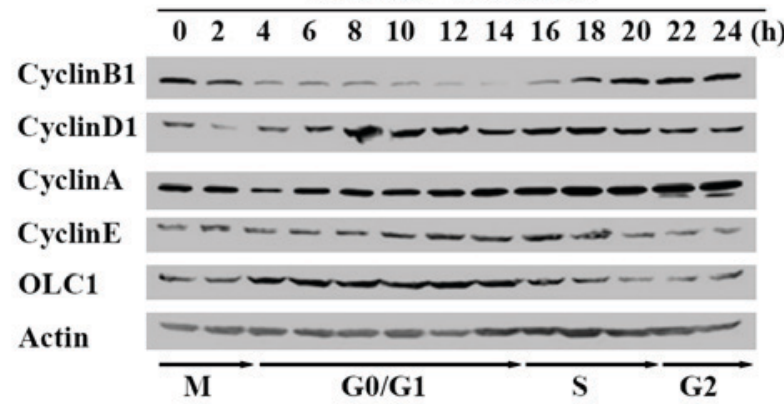

C

Time after nocodazole

$\begin{array}{lllllllllllll}0 & 2 & 4 & 6 & 8 & 10 & 12 & 14 & 16 & 18 & 20 & 22 & 24\end{array}$

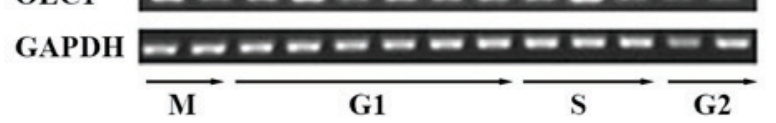

Figure 1. Expression of OLC1 throughout the cell cycle. (A) Using serum starvation, KYSE150/GFP cells were synchronized at the $\mathrm{G}_{0}$ phase Following release, cells were collected every 2 for $24 \mathrm{~h}$. The protein expression of OLC1, cyclin D1, cyclin A and actin was analyzed using western blot analysis. (B) Using $0.4 \mu \mathrm{g} / \mathrm{ml}$ nocodazole, KYSE150/GFP cells were synchronized at the mitotic phase. Following release, cells were collected every 2 for $24 \mathrm{~h}$. The protein expression of OLC1, cyclin B1, cyclin D1, cyclin A, cyclin $\mathrm{E}$ and actin was analyzed using western blot analysis (C) Using reverse transcription polymerase chain reaction, the mRNA expression of OLC1 and GAPDH were detected. OLC1, overexpressed in lung cancer 1.

an increase in OLC1 expression compared with cells treated only with CHX. Similarly, with increasing MG132 treatment times, OLC1 protein expression levels were increased accordingly (Fig. 2B and C). This suggests that OLC1 protein degradation is regulated by the ubiquitin proteasome pathway. Subsequently, immune precipitation as says with an anti-OLC1 antibody was performed to further confirm this. Ubiquitin was detected in the OLC1 immuno complex, and as expected, the amount present was increased following the addition of MG132 (Fig. 2D).

OLC1 protein is degraded by APC/c. The OLC1 protein sequence was analyzed and a D-box motif was identified (amino acids 12-20; Fig. 3A). In the process of ubiquitination and degradation of proteins, D-box motifs are often the recognition sites for the E3 ligase of the APC/c $(7,8)$. Thus, it was hypothesized that the $\mathrm{E} 3$ ligase of $\mathrm{APC} / \mathrm{c}$ may facilitate OLC1 ubiquitination. In the APC/c, APC2 is the most
A

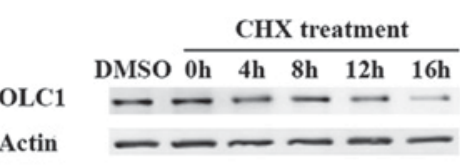

B

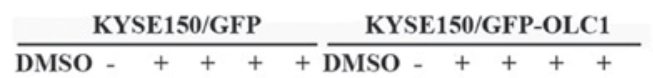

MG132 GFP-OLC1 OLC1 Actin

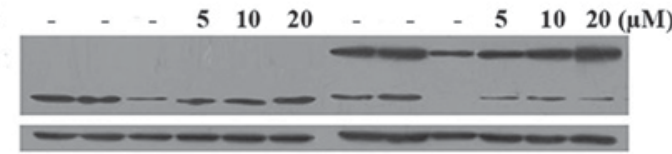

C

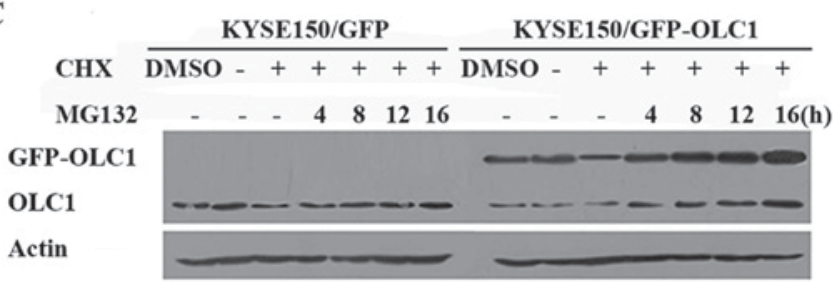

D

MG132 - + +

IP: OLC1 OLC1 Actin

IB:Ubiquitin

IB:OLC1

IB:Actin

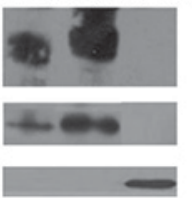

Figure 2. OLC1 protein expression with CHX and MG132 treatment. (A) Following treatment with $100 \mu \mathrm{g} / \mathrm{ml} \mathrm{CHX,} \mathrm{KYSE150/GFP} \mathrm{cells} \mathrm{were}$ collected for western blot analysis of OLC1 protein expression at the indicated times. KYSE150/GFP and KYSE150/GFP-OLC1 cells were co-treated with CHX $(100 \mu \mathrm{g} / \mathrm{ml})$ and MG132 at (B) different concentrations $(5,10$ and $20 \mu \mathrm{M}$ MG132) or (C) for different lengths of time (4, 8, 12 and $16 \mathrm{~h}$ ) with $20 \mu \mathrm{M}$ MG132, with the DMSO, negative control and MG132-negative groups collected for analysis after $16 \mathrm{~h}$ of treatment. Results are representative of three independent experiments. (D) With $(+)$ or without (-) treatment with $20 \mu \mathrm{M}$ MG132, KYSE150/GFP-OLC1 cells were incubated for $16 \mathrm{~h}$, collected and lysed for IP with an anti-OLC1 antibody. Ubiquitins were detected in the immunocomplex, and the presence of OLC1 was verified. IP with an anti-actin antibody was used as a negative control. OLC1, overexpressed in lung cancer 1; CHX, cycloheximide; IP, immunoprecipitation.

important subunit, as it acts as the connector with the target protein. Therefore, the interaction between OLC1 with APC2 was examined. The results demonstrated that OLC1 directly interacts with APC/c through its APC2 component (Fig. 3B), which indicates that OLC1 protein ubiquitination and degradation are mediated by the APC/c pathway.

The activation of APC/c also requires the association with proteins containing tryptophan aspartate. $\mathrm{Cdc} 20$ (termed Fizzy in Drosophila) and Cdh1 (Fizzy-associated in Drosophila) have been identified as two of these activators, and have been demonstrated to activate APC/c E3 ligase and stimulate substrate degradation. These two proteins may directly interact with the APC/c and serve important functions as limiting, substrate-specific activators of APC-dependent proteolysis $(11,12)$. To confirm whether $\mathrm{Cdh} 1$ or $\mathrm{Cdc} 20$ were required for OLC1 protein degradation, Cdh1 and Cdc20 expression was transiently up regulated individually in H1299 cells; it was revealed that the increase of each protein resulted in a reduction in OLC1 protein expression (Fig. 3C). On the contrary, the down regulation of the endogenous Cdh1 or 


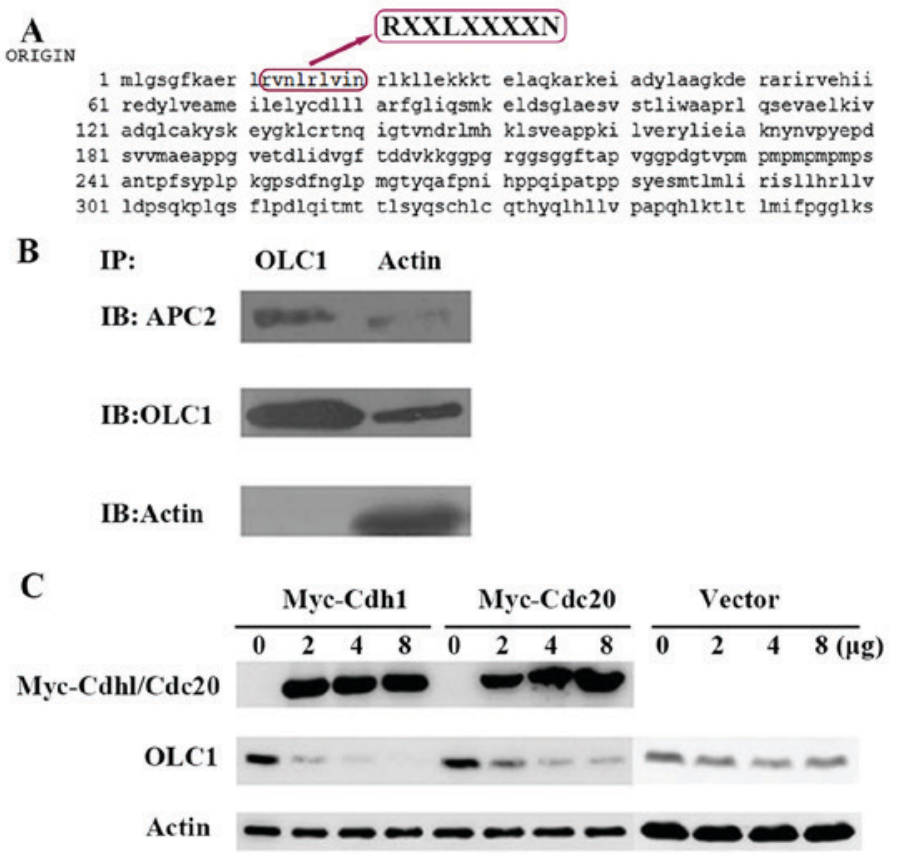

D

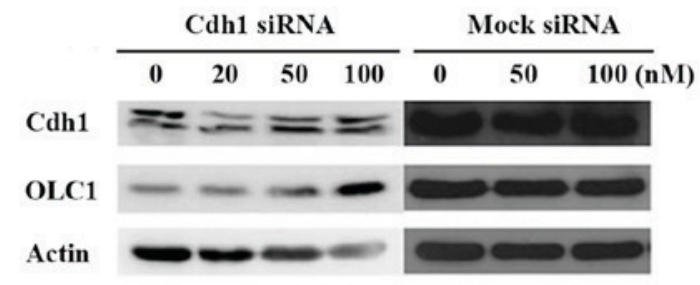

$\mathbf{E}$ Cde20 siRnA Mock siRNA

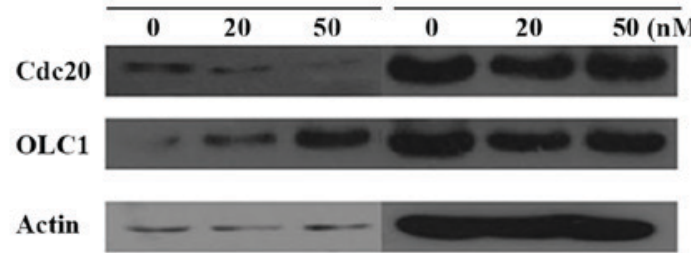

Figure 3. OLC1 protein is degraded by the APC/c. (A) Analysis of the OLC1 protein sequence indicated that it contains a destruction box at the site of amino acids 12-20, which may be recognized by APC/c. (B) To investigate whether OLC1 may bind directly to the components of the APC/c, KYSE150/GFP-OLC1 cells were collected, lysed and subjected to IP. The presence of actin, OLC1 and APC2 in the immunocomplex were verified. (C) Different concentrations $(2,4$ and $8 \mu \mathrm{g})$ of Myc-Cdh1/Cdc20 expression or mock vectors were transiently transfected into H1299 cells for $48 \mathrm{~h}$. Then OLC1, Myc-Cdh1 and Myc-Cdc20 protein expression were evaluated. Actin was included as a loading control. Different concentrations (20,50 and $100 \mathrm{nM})$ of (D) Cdh1 or (E) Cdc20 or mock siRNA were transiently transfected into $\mathrm{H} 1299$ cells for $48 \mathrm{~h}$. Then OLC1, Cdh1 and Cdc20 protein expressions were evaluated. Actin was included as a loading control. OLC1, overexpressed in lung cancer 1; APC/c, anaphase-promoting cyclosome complex; IP, immunoprecipitation; Cdh1, cadherin 1; Cdc20, cell-division cycle protein 20; siRNA, small interfering RNA.

Cdc20 via siRNAs resulted in the increased expression of OLC1 (Fig. 3D and E). From the above results, it was posited that Cdh1 and Cdc20 are involved in the process of OLC1 degradation.

Mutated D-box domains interfere with OLCl protein degradation and accelerate cell growth and colony formation. In the present study, the analysis of the OLC1 protein sequence revealed that it contained a D-box (amino acids 12-20). Generally, substrates containing a D-box or KEN-box domain are recognized by the APC/c E3 ligase, assisted by Cdc20 and Cdh1 $(11,12)$. Therefore, it was hypothesized that the D-box domain mediated the ubiquitination-dependent destruction of OLC1 proteins. The conserved D-box motif was point-mutated, as presented in Fig. 4A, and it was assessed whether this mutation had an effect on OLC1 protein stability. H1299 cells were co-transfected with D-box-mutated GFP-OLC1 (GFP-mut-OLC1) and different concentrations of Cdh1 or Cdc20 expression vectors. As expected, the increased expression of Cdh1 and Cdc20 did not promote the degradation of the mutated OLC1, suggesting that the identified D-box motif was the critical sequence required for OLC1 ubiquitination and the subsequent degradation (Fig. 4B).

Previous studies have demonstrated that OLC1 is an oncogenic protein, as the induced overexpression of OLC1 results in anchorage-independent growth in vitro and malignant transformation in vivo. Additionally, OLC1 has been identified as overexpressed in multiple types of malignant tumor, including in lung cancer and esophageal squamous carcinoma $(1,2)$. Thus, additional experiments were performed to confirm if the cells with mutated OLC1 may exhibit more malignant characteristics. First, a cell growth assay was performed; the growth curve revealed that $\mathrm{H} 1299$ cells expressing OLC1 with a mutated D-box grew significantly faster compared with those expressing wild-type OLC1 (Fig. 4C). This indicated that the D-box mutated OLC1 exhibited a greater capacity to facilitate cell growth. Colony formation assays were also conducted; the cells expressing mutant OLC1 developed a significantly higher number of colonies compared with the control cells (Fig. 4D and E). These results indicated that the mutated D-box motif was not recognized by APC/c E3 ligase, affecting the subsequent degradation of the OLC1 protein. Overall, non-degradable OLC1 presents a greater oncogenic capacity compared with wild-type OLC1.

\section{Discussion}

The OLC1 gene is located in chromosome 16q22.2. When it was first identified in non-lethal yeast mutants in 1999, it was named the IST1 gene (14). In yeast, IST1 participates in the multi vesicular body (MVB) sorting pathway, in which certain membrane proteins are sorted into the lumen of the vacuole for their eventual degradation $(15,16)$. The gene product of IST1/OLC1 in different organisms is highly conserved, including between yeast and humans. In humans, a previous study identified that OLC1 was essential for cytokinesis, another membrane scission event that is topologically similar to MVB formation, and that the depletion of OLC1 resulted in the accumulation of multinucleated cells (7). Cytokinesis is the last stage of the cell cycle, where a cell divides into two 
A

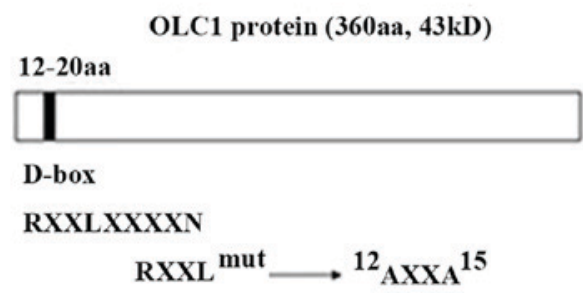

\section{C}

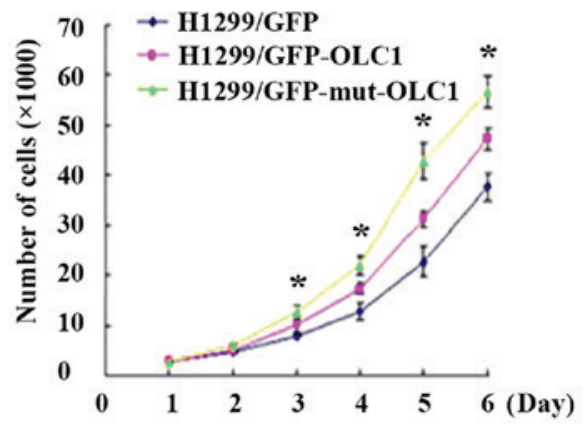

B

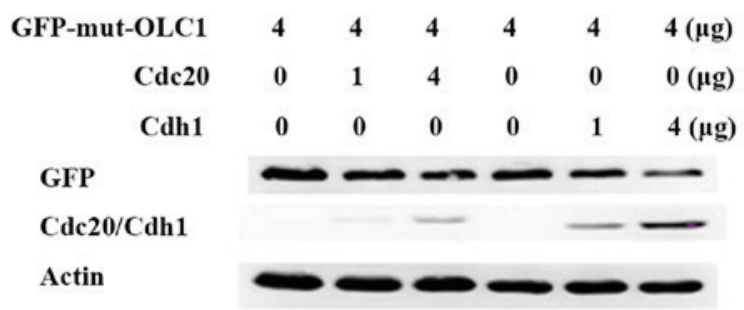

D

H1299/GFP-mut-OLC1

H1299/GFP-OLC1

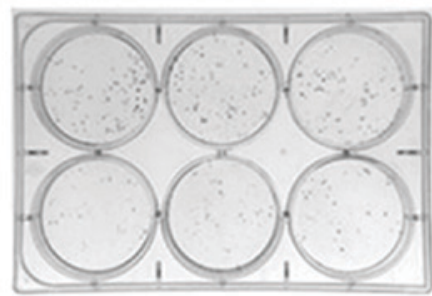

E

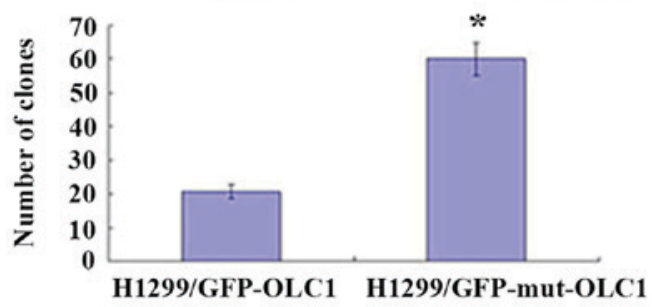

Figure 4. Mutated D-box domains interfere with OLC1 protein degradation and accelerate cell growth and colony formation. (A) A schematic representation of the D-box degradation signal within OLC1. Numbers denote amino acid numbers where the D-box lies, and the conserved sequence motif is annotated. The mutation scheme for the OLC1 D-box is indicated. (B) H1299 cells were co-transfected with $4 \mu \mathrm{g}$ OLC1 (D-box mutant) plasmids and different concentrations of human Myc-Cdh1/Cdc20 expression vectors for $48 \mathrm{~h}$. OLC1, Cdh1 and Cdc20 protein expression were evaluated using western blot analysis. Actin was probed as a loading control. (C) H1299 cells were transfected with $4 \mu \mathrm{g}$ pEGFP-N1, pEGFP-N1-OLC1 or pEGFP-N1-mut-OLC1. Mean values with standard deviations were calculated to produce a cell proliferation curve. The results were obtained in three independent experiments. " $\mathrm{P}<0.05$ vs. wild-type OLC1. (D) A colony formation assay of H1299 cells was performed following transfections of a mutated or wild-typeOLC1 expression vector for $24 \mathrm{~h}$ and (E) the number of colonies were counted. The results were obtained from three independent experiments. " $\mathrm{P}<0.05$ vs. control cells. OLC1, overexpressed in lung cancer 1; D-box, destruction box; Cdh1, cadherin 1; Cdc20, cell-division cycle protein 20.

daughter cells, passing the same amount of genetic material to each daughter cell. Therefore, abnormal cytokinesis will result in the uneven distribution of the chromosomes in the cell, inducing cell genome instability and potentially leading to the development of a tumor $(17,18)$.

A previous study regarding OLC1 studied it from another angle; the OLC1 gene was identified as a potential oncogene, that was highly expressed in lung cancer, in 2008 (1). It was identified that the over expression of OLC1 was associated with smoking history in patients with lung cancer, and that this overexpression induced tumor formation in athymic mice, whereas the knockdown of OLC1 increased apoptosis and decreased colony formation. It was also revealed that cigarette smoke may increase OLC1 protein expression at the cellular level (8). Previous studies have revealed that the OLC1 protein is highly expressed in numerous malignant tumor types, including esophageal squamous cancer, colorectal cancer, breast cancer and ovarian cancer. Furthermore, high expression levels of OLC1 are associated with a poor prognosis in a number of these cancer types $(2,19-21)$.

However, until the present study, few studies focused on the molecular regulatory mechanism controlling OLC1 expression. Given the critical function of OLC1 from yeast to humans, studying how OLC1 is regulated may provide further insight into human tumor genesis. In the present study, it was demonstrated that OLC1 protein may be cell-cycle-dependent with a short half-life, and that it may be degraded through the APC/c-mediated proteasome pathway.

Two approaches for cell cycle synchronization were conducted in order to study the expression pattern of OLC1 during the entire cell cycle. Given that the expression of numerous other cyclins fluctuates in different phases of the cell cycle, cyclins A, B1, D1 and E were examined to determine the dynamic changes in OLC1 expression in the cell cycle. The OLC1 protein exhibited higher expression levels during the $G_{0} / G_{1}$ phase compared with the other phases, whereas the mRNA expression levels of OLC1 demonstrated little change across the entire cell cycle. In conclusion, the expression of OLC1 is regulated through post-transcriptional mechanisms during the cell cycle.

Mammalian cells contain two distinct major proteolytic pathways. One important non-lysosomal mechanism for the degradation of intracellular proteins is the ubiquitin-proteasome pathway $(22,23)$. Ubiquitin and ubiquitin ligase post-translationally modify the abundance of target proteins, including oncogenes or tumor suppressor genes, and thereby alter their effect. For example, the Akt signaling pathway has many important biological functions, while its deregulation is associated to the development of numerous types of malignant tumors in humans. Although previous studies have primarily 
focused on Akt phosphorylation, other post-translational modifications to Akt, including ubiquitination, have been demonstrated to serve an important function in Akt activation. A previous study revealed that a cancer-associated Akt mutation within the Akt PH domain (E17K) was identified in a range of human cancer types, including colon and breast cancer. Akt E17K mutants displayed enhanced Akt ubiquitination, contributing to Akt hyperactivation and constitutive Akt membrane recruitment, suggesting a potential role for Akt ubiquitination in cancer (24). MDM2 is an E3 ubiquitin ligase with strong clinical relevance due to its ability to regulate the tumor suppressor p53. By recruiting an E2 ubiquitin-conjugating enzyme, MDM2 facilitates the export of p53 from the nucleus to the cytoplasm, and targets p53 for ubiquitin-dependent proteasome degradation (25).

Similar to the processes revealed for other oncogenes and tumor suppressor genes, ubiquitin-mediated proteolysis was determined as an important regulator of OLC1 protein expression. In the present study, it was demonstrated that the expression of OLC1 was elevated following treatment with MG132, a proteasome inhibitor. OLC1 protein degradation decreased, and increased ubiquitin was ligated to OLC1 proteins following MG132 treatment. All these results indicate that OLC1 degradation must be regulated by the ubiquitin-proteasome pathway.

A previous study has demonstrated that the interactions between OLC1 and other proteins are involved in a range of biological processes, including MVB biogenesis, cytokinesis and enveloped virus budding (16). In mammalian cells, the complete function of OLC1 is required for efficient abscission during cytokinesis (5). Additionally, in the process of cytokinesis, the E3 ligase APC/c mediates the ubiquitin-dependent proteolysis of cell-cycle-regulating proteins $(26,27)$. Thus, we hypothesized that APC/c may function as an E3 ligase for OLC1 ubiquitination. In a further study, it was revealed that there was a conserved D-box motif of RVNLRLVINR within the OLC1 protein sequence, which is a conserved and well-recognized site for E3 ligase APC/c in a number of ubiquitinated substrates (28). Additionally, co activators containing tryptophan aspartate are also required to assist the activation of APC/c. Two of these proteins have been identified as Cdc20 and Cdh1 (13,29). Usually, Cdc20 targets D-box-containing substrates, whereas Cdh1 may interact with either the D-box or KEN box of proteins $(11,30)$. Consistent with this, in the present study, immune precipitation as says revealed that OLC1 may directly bind to APC/c through the subunit APC2, and either Cdc20 or Cdh1 up regulation induced decreased OLC1 expression. Likewise, cells with Cdc20 and Cdh1 down regulated via siRNA exhibited OLC1 protein stabilization. Furthermore, mutations to the OLC1 D-box significantly reduced OLC1 degradation. Collectively, it was confirmed that the APC/c mediated ubiquitin-proteasome pathway regulated the degradation of OLC1.

Using constructed wild-type and D-box-mutated OLC1 plasmids, functional experiments were performed. Growth curve and colony formation assays demonstrated that the overexpression of the non-degradable OLC1 protein not only facilitated cell growth, but also enhanced the clone-forming capability of the cells. These findings reveal that the destruction of the degradation domain results in the abnormal accumulation of the OLC1 mutant, which could not be degraded through the APC/c-mediated ubiquitin-proteasome pathway. These malignant cell phenotypes support the idea that OLC1 exhibits on cogenic properties.

In conclusion, these findings have the potential to make important contributions in clarifying the mechanism of the APC/c-mediated destruction of the candidate oncogene OLC1.

\section{Acknowledgements}

The authors would like to thank Dr Shimada of Kyoto University (Kyoto, Japan) for providing the KYSE150 cells and Professor Shujun Cheng of the Chinese Academy of Medical Sciences and Peking Union Medical College, Cancer Institute and Cancer Hospital (Beijing, China), for providing the pEGFP-N1-OLC1 and pEGFP-N1 plasmids. pCMV-Myc-Cdh1 and pCMV-Myc-Cdc20 plasmids were generously provided by Professor James Hsieh of Washington University (Seattle, WA, USA).

\section{Funding}

This research was supported by Grants from the National High Technology Research and Development Program of China (grant no., 2006AA02A403).

\section{Availability of data and materials}

All data generated or analyzed during this study are included in this published article.

\section{Author contributions}

QMZ and TT obtained funding and participated in the study coordination. ZXJ and WC were responsible for study design. ZXJ, WC and NY performed the experimental procedures. LJD and MF performed data analysis, and ZXJ, WC and TT were responsible for editing and review of the manuscript. TT was also responsible for study design.

\section{Ethics approval and consent to participate}

Not applicable.

\section{Consent for publication}

Not applicable.

\section{Competing interests}

The authors declare that they have no competing interests.

\section{References}

1. Yuan J, Ma J, Zheng H, Shi T, Sun W, Zhang Q, Lin D, Zhang K, He J, Mao Y, et al: Overexpression of OLC1, cigarette smoke, and human lung tumorigenesis. J Natl Cancer Inst 100: 1592-1605, 2008.

2. Li X, Suo J, Shao S, Xue L, Chen W, Dong L, Shi J, Fu M, Lu N, Zhan Q and Tong T: Overexpression of OLC1 promotes tumorigenesis of human esophageal squamous cell carcinoma. PLoS One 9: e90958, 2014.

3. Dimaano C, Jones CB, Hanono A, Curtiss M and Babst M: Ist1 regulates Vps4 localization and assembly. Mol Biol Cell 19: 465-474, 2008 
4. Rue SM, Mattei S, Saksena S and Emr SD: Novel Ist1-Did2 complex functions at a late step in multivesicular body sorting. Mol Biol Cell 19: 475-484, 2008.

5. Agromayor M, Carlton JG, Phelan JP, Matthews DR, Carlin LM, Ameer-Beg S, Bowers K and Martin-Serrano J: Essential role of hIST1 in cytokinesis. Mol Biol Cell 20: 1374-1387, 2009.

6. Bajorek M, Schubert HL, McCullough J, Langelier C, Eckert DM, Stubblefield WM, Uter NT, Myszka DG, Hill CP and Sundquist WI: Structural basis for ESCRT-III protein autoinhibition. Nat Struct Mol Biol 16: 754-762, 2009.

7. Bajorek M, Morita E, Skalicky JJ, Morham SG, Babst M and Sundquist WI: Biochemical analyses of human IST1 and its function in cytokinesis. Mol Biol Cell 20: 1360-1373, 2009.

8. Zhang X, Xiao T, Cheng S, Tong T and Gao Y: Cigarette smoke suppresses the ubiquitin-dependent degradation of OLC1. Biochem Biophys Res Commun 407: 753-757, 2011.

9. Castro A, Bernis C, Vigneron S, Labbe JC and Lorca T: The anaphase-promoting complex: A key factor in the regulation of cell cycle. Oncogene 24: 314-325, 2005.

10. ManchadoE,EgurenMandMalumbresM:Theanaphase-promoting complex/cyclosome (APC/C): Cell-cycle-dependent and -independent functions. Biochem Soc Trans 38: 65-71, 2010.

11. da Fonseca PC, Kong EH, Zhang Z, Schreiber A, Williams MA, Morris EP and Barford D: Structures of APC/C $(\mathrm{Cdh} 1)$ with substrates identify Cdh1 and Apc10 as the D-box co-receptor. Nature 470: 274-278, 2011.

12. Robbins JA and Cross FR: Regulated degradation of the APC coactivator Cdc20. Cell Div 5: 23, 2010.

13. Zhang S, Chang L, Alfieri C, Zhang Z, Yang J, Maslen S, Skehel M and Barford D: Molecular mechanism of APC/C activation by mitotic phosphorylation. Nature 533: 260-264, 2016.

14. Entian KD, Schuster T, Hegemann JH, Becher D, Feldmann H, Güldener U, Götz R, Hansen M, Hollenberg CP, Jansen G, et al: Functional analysis of 150 deletion mutants in Saccharomyces cerevisiae by a systematic approach. Mol Gen Genet 262: 683-702, 1999

15. Allison R, Lumb JH, Fassier C, Connell JW, Ten Martin D, Seaman MN, Hazan J and Reid E: An ESCRT-spastin interaction promotes fission of recycling tubules from the endosome. J Cell Biol 202: 527-543, 2013

16. Guo EZ and Xu Z: Distinct mechanisms of recognizing endosomal sorting complex required for transport III (ESCRT-III) protein IST1 by different microtubule interacting and trafficking (MIT) domains. J Biol Chem 290: 8396-8408, 2015.
17. D'Avino PP, Giansanti MG and Petronczki M: Cytokinesis in animal cells. Cold Spring Harb Perspect Biol 7: a015834, 2015.

18. Tormos AM, Taléns-Visconti R and Sastre J: Regulation of cytokinesis and its clinical significance. Crit Rev Clin Lab Sci 52: 159-167, 2015.

19. Jia C, Li X, Sun H and Sui L: Over-expression of the overexpressed in lung cancer 1 is associated with poor prognosis in epithelial ovarian cancer. J Surg Oncol 107: 847-852, 2013.

20. Liu J, Song H, Yao L, Liu Y, Zhang Y, Zhao H, Ji H and Wang Y: Over-expression of the overexpressed in lung cancer-1 is associated with poor prognosis in colorectal cancer. Anticancer Res 34: 367-372, 2014.

21. Ou-Yang QH, Duan ZX, Jin Z and Lei JX: OLC1 is overexpressed in breast cancer and its expression correlates with poor patient survival. Tumour Biol 35: 8823-8827, 2014.

22. Chondrogianni N, Petropoulos I, Grimm S, Georgila K, Catalgol B, Friguet B, Grune T and Gonos ES: Protein damage, repair and proteolysis. Mol Aspects Med 35: 1-71, 2014

23. Jain CK, Arora S, Khanna A, Gupta M, Wadhwa G and Sharma SK: The ubiquitin-proteasome pathway an emerging anticancer strategy for therapeutics: A patent analysis. Recent Pat Anticancer Drug Discov 10: 201-213, 2015.

24. Yang WL, Wu CY, Wu J and Lin HK: Regulation of Akt signaling activation by ubiquitination. Cell Cycle 9: 487-497, 2010.

25. Chao CC: Mechanisms of p53 degradation. Clin Chim Acta 438 139-147, 2015

26. Lindon C: Control of mitotic exit and cytokinesis by the APC/C Biochem Soc Trans 36: 405-410, 2008.

27. Chang L and Barford D: Insights into the anaphase-promoting complex: A molecular machine that regulates mitosis. Curr Opin Struct Biol 29: 1-9, 2014.

28. Barford D: Structure, function and mechanism of the anaphase promoting complex (APC/C). Q Rev Biophys 44: 153-190, 2011.

29. Van Voorhis VA and Morgan DO: Activation of the APC/C ubiquitin ligase by enhanced E2 efficiency. Curr Biol 24: 1556-1562, 2014.

30. Cho HJ, Lee EH, Han SH, Jeong JH, Kwon J and Kim H: Degradation of human RAP80 is cell cycle regulated by Cdc20 and Cdh1 ubiquitin ligases. Mol Cancer Res 10: 615-625, 2012. International (CC BY-NC-ND 4.0) License. 